\title{
The Nature Event's Probabilistic Risk Assessment of Nuclear Power Plants with Improvements
}

\author{
CHEN Yan, YU Shaoqing, ZHANG Chunming, CHAI Jianshe* \\ Nuclear and Radiation Safety Center, Ministry of Environmental Protection \\ Beijing, 100082, China \\ *E-mail: chaijshe@163.com \\ Received 31 December 2011 \\ Accepted 14 March 2012
}

\begin{abstract}
Probabilistic risk assessment is the systematic and comprehensive methodology to evaluate the risk of nuclear power plants. Currently some aspects, which are the hazard analysis and fragility evaluation in the probabilistic risk assessment of the nature event in nuclear power plants need to be improved. This paper introduces the principle of risk assessment of the nature disaster, and studies the relations between the two risk analysis methods, finally discusses the application of the technologies, which are the hazard factor, vulnerability analysis, loss risk and risk zone map in the nature disaster's risk assessment, in the nature event PRA of nuclear power plants.
\end{abstract}

Keywords: PRA, risk assessment of nature disaster, hazard analysis, fragility evaluation.

\section{一种改进的核电站自然灾害风险评价方法}

陈妍, 余少青, 张春明, 柴建设

环境保护部核与辐射安全中心, 北京 100082

\begin{abstract}
摘要: 概率风险技术是核电站风险定量评价常用的方法, 目前核电站自然灾害概率风险评价方法在危害性 分析、脆弱性评估等方面函需补充完善。本文引入了自然灾害风险分析的基本原理, 研究了自然灾害风险 分析的基本原理与核电站自然灾害概率风险评价方法的联系, 探讨了其在核电站自然灾害风险中的危害 性、脆弱性、损失分析和风险区划图方面的借鉴。该方法在完善核电站自然灾害概率风险评价方面能发挥 重要作用。
\end{abstract}

关键词：PRA, 自然灾害风险分析, 危害性, 脆弱性

\section{1. 引言}

二十世纪自然灾害在全球范围内造成了几百万 人的死亡，在我国仅因地震造成的人员死亡约 56 万 人。二十一世纪以来全球自然灾害频发,印度洋海 啸、美国卡特里那风具风、汶川地震、海地地震、澳 大利亚洪灾、日本大地震海啸等自然灾害给人类造 成了灾难性的损失。联合国国际减灾战略发布的全 球自然灾害统计报告指出, 在 2000 年至 2009 年 间, 全球共发生自然灾害事件 3852 起, 直接导致超 过 78 万人死亡, 近 20 亿人受到影响, 经济损失高 达 9600 亿美元。

目前全球共有 440 多座在运核电站, 各核电站 虽然在设计中充分考虑了防御自然灾害的能力, 采
取了预防和缓解超设计基准事故的措施, 但是自然 灾害仍然会对核电站造成一定风险。1992 年的安德 鲁风具风使得迈阿密南部的土耳其角核电站断电, 电 站的应急柴油发电机确保了设备正常运行; 2007 年 日本中越冲地震, 引起柏崎刚羽核电厂 3 号机组主 变着火、6 号机乏燃料水池的水溅出、固体废物捾 存罐破损, 致使微量放射性物质泄漏; 2011 年日本 9.0 级大地震及其引起的海啸造成福岛核电站的严重 损坏, 导致大量放射性物质释放。国际原子能机构

（IAEA）在对福岛核事故的调查报告中指出对于超 设计基准的自然灾害, 自然事件概率风险评价方法 的研究有助于核电站的外部灾害风险评估和事故管 理措施的改善 ${ }^{[1]}$ 。 
概率风险评价（Probabilistic Risk Assessment, PRA）是核电站风险定量评价常用的方法。PRA 方 法首次大规模应用于核电站的安全研究是 1975 年的

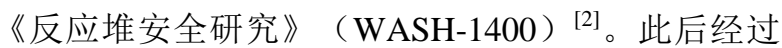
三十多年的发展和完善, PRA 已经被公认为核电站 安全分析的有效工具。IAEA 指出 “PRA 技术已经 成为核电站安全评价的一个标准化工具, 使人们能 够深入地了解核电站的设计、性能和环境影响, 包 括对支配性风险因素的鉴别以及对可降低风险的各 种方案进行比较。PRA 为安全有关问题的决策提供 了协调一致、完整的方法”。核电站 PRA 按始发事 件范围可以分为内部事件和外部事件的 PRA, 外部 事件 PRA 进一步分为自然事件和人为事件的 PRA。

核电站自然事件 PRA 是一种对地震、外部火 灾、台风、洪水等自然灾害造成电站风险的定量评 价方法, 其在内部事件概率风险评价模型的基础上 加入自然灾害造成的影响。核电站自然事件 PRA 主 要包括自然灾害的危害性分析、脆弱性评估和系统 分析及定量化三部分内容。1988 年美国核管会 （NRC）要求各核电站对严重事故的易损性进行检 查评价, 其中外部事件的单个电厂检查 (IPEEE) 推动了自然事件概率风险评价的发展。1991 年 NUREG-1407 总结出进行 IPEEE 的核电站的外部事 件造成的堆芯损伤频率 (CDF) 在 $10^{-6}-10^{-4}$ 堆 - 年的 范围, 与内部事件造成的 CDF 在同一量级。我国 1984 年开始研究秦山和大亚湾核电站的概率风险评 价方法, 目前国内核电站一级内部事件 PRA 已经基 本完成。外部事件 PRA 的研究自 2008 年初步开 展, 在自然灾害的危害性分析和脆弱性评估等方面 亟需与各学科加强合作研究, 以补充完善风险评价 方法中的数据和分析方法, 推进我国核电站自然灾 害风险评估工作。

核电站自然事件 PRA 属于自然灾害风险分析的 范畴。自然灾害风险分析是对风险区遭受不同强度 自然灾害的可能性及其可能造成的后果进行定量分 析和评估, 其能够科学认识自然灾害的孕育、发 生、发展和可能造成的影响, 避免防灾减灾行动的 盲目性, 是防灾减灾的重要内容之一 ${ }^{[3]}$ 。自然灾害 风险分析自联合国 1987 年提出 “国际减轻自然灾害 十年” 以来得到了进一步的研究发展, 其重要内容 包括致灾因子分析、承灾体易损性评价、灾情损失 分析和减灾对策等方面。目前国内已有较为系统地 自然灾害风险评价和风险分析的理论和方法体系 ${ }^{[3,4]}$, 但自然灾害风险分析理论和技术发展仍较缓慢 ${ }^{[5]}$ 。

基于自然灾害的多因性、系统性和不可预期 性, 需要综合多个学科的技术来减轻自然灾害造成 的损失 ${ }^{[6]}$ 。同时，基于核电站放射性危害的特殊 性, 需要结合目前的 PRA 技术和自然灾害风险分析
原理与实践对自然灾害造成的核电站风险进行深入 研究, 以减轻可能的放射性后果。因此, 本文将回 顾自然灾害风险分析的基本原理和核电站的自然事 件概率风险评价方法, 并以地震灾害为例研究两种 风险分析方法的关键技术要素的联系，探讨自然灾 害风险分析的基本原理在核电站自然灾害风险中的 危害性、脆弱性和损失分析方面的借鉴。

\section{2. 自然灾害风险分析的基本原理 ${ }^{[3,7]}$}

自然灾害风险分析的基本原理是正视自然灾害 系统本身所固有的复杂性和不确定性，从基本的元 素着手分析, 对其进行组合, 进行不确定性意义下 的量化分析。自然灾害风险分析的基本内容主要包 括致灾因子风险分析、承灾体易损性评价、灾情损 失分析和减灾对策四个方面内容, 其关系如图 1 。

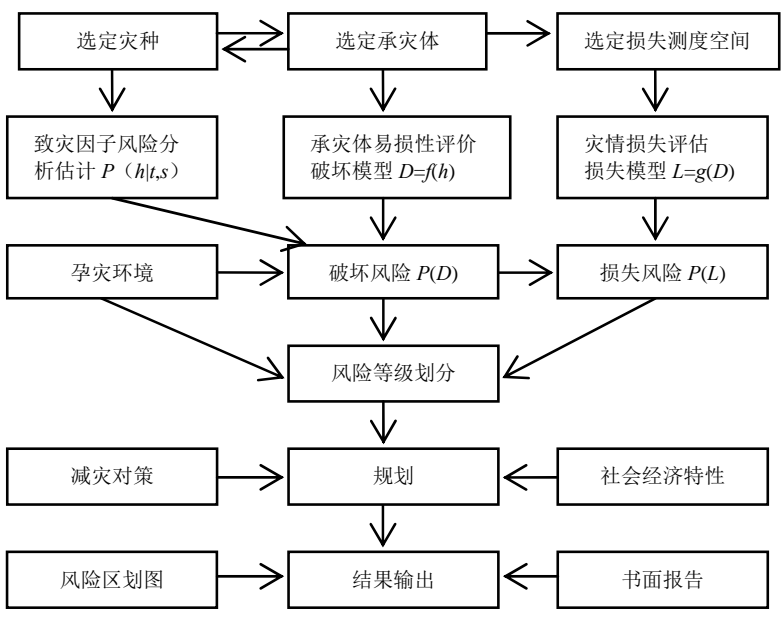

图 1. 自然灾害风险分析流程图 ${ }^{[3]}$

（1）致灾因子风险分析

可能造成灾害的因素称为致灾因子。在自然灾 害系统中，致灾因子通常指地震、洪水、干旱等灾 源。致灾因子风险分析主要研究给定区域内各种强 度的自然灾害发生的可能性, 即估计时段 $t$ 内, 在 给定区域 $s$ 内, 致灾因子以 $h$ 强度发生的条件可能 性 $P(h \mid t, s)$ 。通常说的地震危害性分析、洪水风险实 质上都是致灾因子风险分析。

（2）承灾体易损性评价

承受灾害的对象称为承灾体。承灾体是复杂的 能量转化系统, 担当着将自然灾害的破坏性能量转 化为破坏现象的角色。承灾体易损性评价的核心是 根据给定的致灾因子强度 $h$, 计算承灾体破坏程度 $D$ 的破坏模型 $D=f(h)$, 主要包括以下三方面内容:

风险区确定：研究一定强度自然灾害发生时的 受灾范围。 
风险区特性评价：对风险区内主要建筑物、其 它固定设备和建筑内部财产, 风险区内的人口数 量、分布和经济发展水平等进行分析和评价。

抗灾性能分析：对风险区内的财产进行抗灾性 能分析（也称脆弱性分析）。许多承灾体的破坏机 理还没有完全掌握, 函数关系 $f(h)$ 通常使用实验模 拟、历史灾情统计回归、模糊关系矩阵等方法进行 不确定的脆弱性分析。

\section{（3）灾情损失评估}

灾情损失评估指评估风险区内一定时段内可能 发生的一系列不同强度自然灾害给风险区造成的可 能后果。损失包括经济损失和人员伤亡损失。灾情 损失评估的核心是更具破坏程度 $D$ 计算损失程度模 型 $L=g(D)$ 。自然灾害系统几乎不可能用物理模拟的 方式寻找关系、更多地是用统计方法并用专家经验 来加以评估。

\section{(4) 减灾对策}

减灾对策是为减轻自然灾害的损失或影响程度 而采取的对策。如对建筑物进行抗震设计、制定紧 急救灾预案、建立巨灾保险基金等。减灾风险分析 需要评估, 如果采取了某种减灾对策 $f$, 当发生强度 为 $h$ 的自然灾害打击时, 造成额外损失 $l$ 的可能性 $p(l l f, h)$ 。

综合自然灾害风险分析的主要内容, 可以看到 自然灾害风险分析需要由各灾种的专家提供给定区 域内自然致灾因子发生时、空、强的可能性数值, 由防灾减灾工程师依据致灾因子强度, 提供人类社 会系统各种破坏的可能性数值, 由经济学家和社会 学家们依据破坏程度, 推测各种损失的可能性数 值, 最后, 由自然灾害风险分析人员将三个环节的 可能性数值组合起来，给出损失风险。

\section{3. 核电站自然事件 PRA 方法}

PRA 是以概率论为基础、事件树/故障树为工具 的风险定量评价技术。核电站概率风险评价以真实 而非保守的方式将电站所有有关的信息, 包括设 计、建造、运行、维修、设备可靠性、人因可靠 性、事故进程等综合考虑到一个电站 PRA 模型中, 评价电站对公众健康与安全的潜在影响。核电站 PRA 能够定量评估核电站风险, 给出构筑物、系 统、部件（SSC）的相对风险及重要度排序, 进而 发现电站的薄弱环节, 并有效配置资源进行管理和 控制风险。

PRA 一般分为三级 ${ }^{[8]}$, PRA 的研究内容框架如 图 2。其中一级 PRA 的目的是计算堆芯损坏频率。 二级 PRA 是在一级的分析基础上研究堆芯损坏后的 事故进程及安全壳响应, 评价各种放射性核素向环 境的释放量及释放频率, 该级可以分析减缓堆芯损
坏后的事故后果途径和提出事故管理的具体意见。 三级 PRA 是对二级 PRA 结果加上厂外后果进行评 价进一步研究放射性物质在环境中的扩散及其对公 众和环境的影响, 三级 PRA 能够对后果减缓措施的 相对重要性做出分析, 也能对应急响应计划的制定 提供支持。自然事件 PRA 是在内部事件 PRA 模型

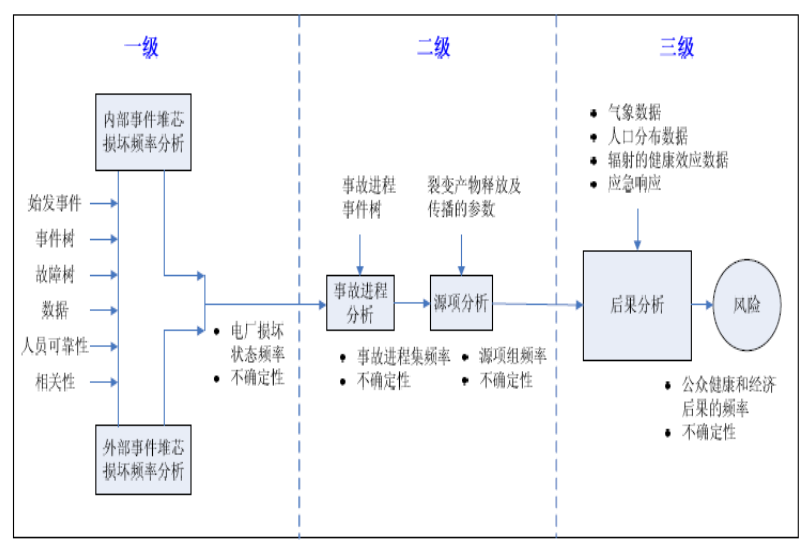

图 2. 核电站 PRA 的内容框架

基础上加入由自然灾害造成的影响, 如危害性分析 为自然灾害造成的始发事件频率提供数据, 脆弱性 分析为始发事件和系统分析中设备失效提供数据 等。自然事件 PRA 主要包括三方面内容: 自然灾害 的危害性分析 (Hazard analysis)、脆弱性评估 （Fragility evaluation）和系统分析及定量化。

\section{4. 自然灾害风险评价方法的联系}

核电站自然事件 PRA 属于自然灾害风险分析的 范畴, 在研究方法内容上与自然灾害风险分析多有 相似之处。本节以地震 PRA 为例 ${ }^{[9,10]}$, 比较这两种 方法的关键技术要素, 探讨关键要素之间的联系。 地震 PRA 的研究方法流程如图 3, 其关键技术要素 是地震危害性分析、地震脆弱性评估、电厂系统和 事件序列分析及堆芯损坏频率定量化评估。

\section{1. 地震概率风险分析}

\section{（1）地震危害性分析}

核电站地震危害性分析的目的是研究核电站所 在厂址的不同水平地震地面运动 (如峰值地面加速 度, PGA ) 的发生频率。核电站地震危害性分析的 步骤是首先确定研究区域的地震源, 然后统计该区 域的地震历史数据评估不同震级/烈度的地震发生频 率, 研究衰减关系以评估地震导致的厂址的地面运 动的强度, 最后综合上述信息评估研究区域的地面 运动参数超出某值的超越频率。地震危害性分析的 结果是描述某厂址不同水平地面运动发生频率的曲 
线图, 通常用 $H(g)$ 表示, 如图 4, 其不确定主要来 自衰减关系、地震源地质构造和可能发震强度的假 设。

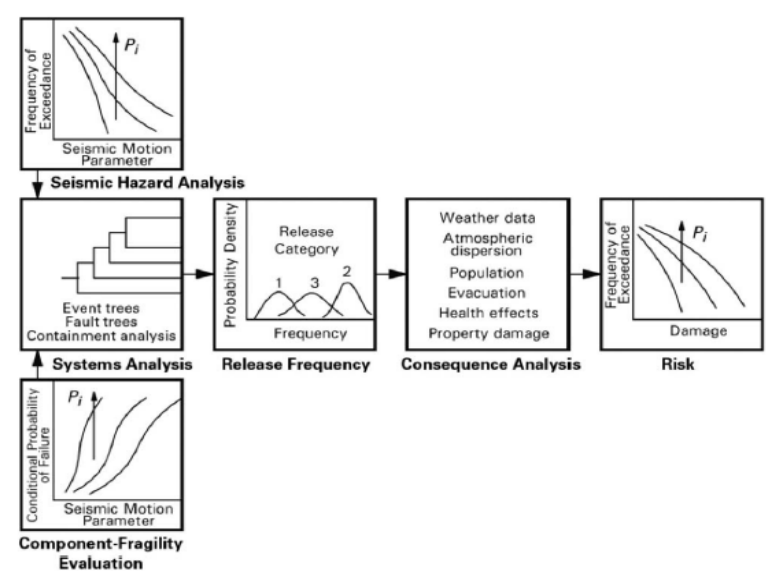

图 3. 地震 PRA 的研究方法流程 ${ }^{[10]}$

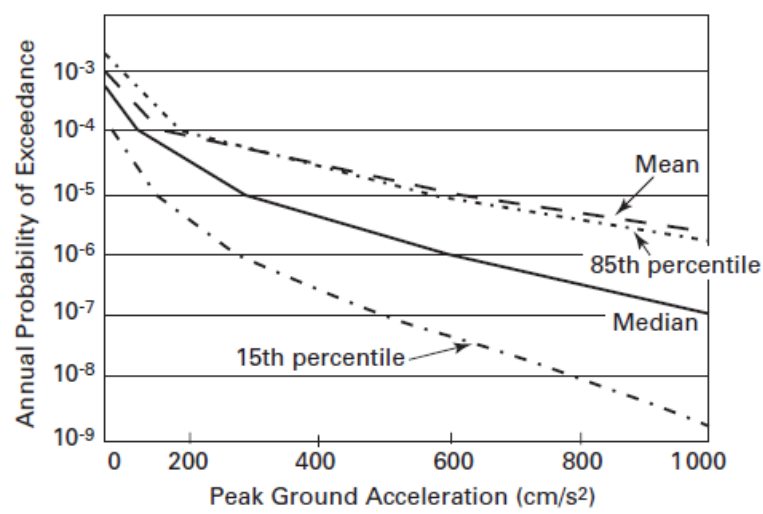

图 4. 地震危害性曲线 ${ }^{[10]}$

\section{（2）地震脆弱性评估}

地震脆弱性评估目的是评估电厂重要系统、构 筑物和部件在各级别的地面运动影响下 (给定地震 动参数, 如 $P G A$ 、强度、加速度谱值) 的条件失效 概率, 其反映了地震导致的始发事件频率（如应急 交流电源丧失, 强迫循环冷却系统丧失）以及不同 缓解系统或设备的条件失效概率 (如辅助给水系 统）。地震脆弱性评估需要电厂设计基准、设计阶 段 SSC 的响应分析以及材料特性等, 同时电厂巡访 对于地震脆弱性评估也非常重要。评估结果是描述 SSC 在不同 PGA 下的条件失效概率的曲线图, 如图 5 。核电站中部件的脆弱性曲线 ${ }^{[11,12,13]}$ 通常用 Eq.(1) 描述:

$$
F(a, Q)=\Phi\left[\frac{\ln \frac{a}{A_{m}}+\beta_{U} \Phi^{-1}(Q)}{\beta_{R}}\right] .
$$

其中, $\Phi$ 为标准高斯累积分布函数, $\Phi^{-1}$ 是其逆函 数。 $A_{m}$ 是地面运动参数中位数, $\beta_{U}$ 描述模型不确 定性（认知不确定性）, $\beta_{R}$ 描述随机不确定性（地 震及其影响的不确定性）, $Q$ 为曲线的置信度, 取 值 $(0,1), a$ 是地面运动强度参数。

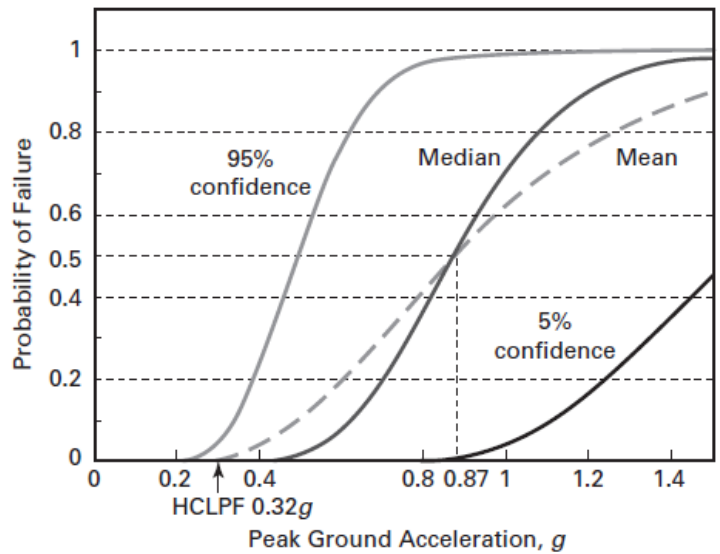

图 5. 地震脆弱性评估曲线 ${ }^{[10]}$

（3）系统/事故序列分析和定量化

系统/事故序列分析将综合电厂逻辑图、部件脆 弱性评估和地震危害性分析, 对导致并传递地震堆 芯损坏事故序列的各种构筑物和设备失效的组合进 行建模, 然后对事故序列频率进行定量化。即危害 性分析为始发事件频率提供数据, 脆弱性分析为始 发事件和系统分析中设备失效提供数据, 继而通过 始发事件、事件序列、故障树和相关性等分析，使 用软件建立核电站 PRA 模型进而得到堆芯损坏频率 定量化结果。堆芯损坏频率实质上是由灾害性曲线 和脆弱性曲线卷积成的一族数值 $\left\{<p_{i j,}, f_{i j}>\right\}$ 。其中, $f_{i j}$ 是地震造成的电站损坏频率, $p_{i j}$ 是这种损坏频率的 可能性:

$$
\begin{gathered}
\mathrm{p}_{\mathrm{ij}}=q_{i} p_{j} . \\
f_{i j}=\int_{0}^{\infty} F_{i}(a) \frac{d H_{j}}{d a} d a .
\end{gathered}
$$

其中， $H_{j}$ 代表第 $j$ 个危害性曲线， $F_{i}$ 代表第 $i$ 个电厂 脆弱性曲线; $q_{i}$ 是第 $i$ 个脆弱性曲线的可能性, $p_{j}$ 是 第 $j$ 个危害性曲线的可能性 ${ }^{[10,11,14]}$ 。

\section{2. 自然灾害原理与地震 PRA 方法的联系}

基于地震 PRA 属于自然灾害分析原理的范畴, 可以把地震 PRA 中的技术要素纳入自然灾害风险分 析的基本原理框架, 如图 6。可以看到, 两种风险 分析方法中的技术要素有许多有相似之处, 如致灾 因子、易损性评价、破坏风险方面是等价的; 损失 
风险、孕灾环境、减灾对策方面原理上一致，但范 围不同。

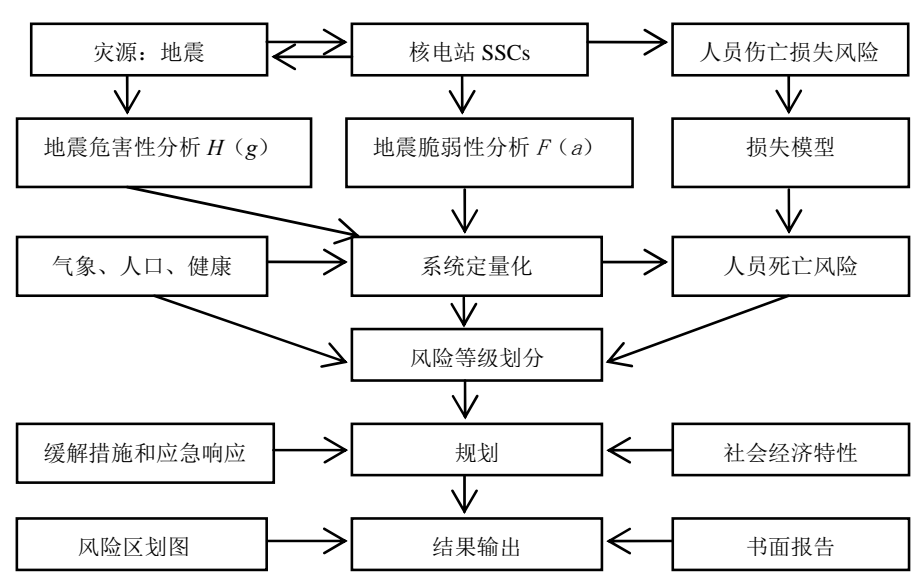

图 6 地震 PRA 与自然灾害风险分析基本原理的关系图

a) 致灾因子风险分析与危害性分析：致灾因子 风险分析主要研究给定区域内各种强度的自然灾害 发生的可能性, 核电站地震危害性分析主要研究核电 站所在厂址的不同水平地震地面运动的发生频率, 因 此从统计角度致灾因子发生的条件可能性 $P(h \mid t, s)$ 与 核电站厂区地震危害性曲线 $H(g)$ 相对应, 即地震危 害性分析实质上是致灾因子 (地震) 风险分析。地 震危害性曲线为核电站地震 PRA 的始发事件频率提 供输入数据, 因此危害性分析环节需要 PRA 人员提 出需求, 地震地质专家提供数据信息。

b) 承灾体易损性评价与核电站地震脆弱性评 价：承灾体易损性评价是计算承灾体在给定致灾因 子强度下的破坏程度, 核电站地震脆弱性评价是评 估 SSC 在各级别的地面运动影响下的条件失效概 率。因此, 核电站中的 SSC 是自然灾害原理中的承 灾体, 破坏程度 $D=f(h)$ 与失效概率 $F(a)$ 等价描述, 承灾体（SSC）易损性评价与核电站地震脆弱性评 价一致。基于 SSC 失效概率是地震导致的始发事件 频率以及不同缓解系统或设备的条件失效概率的输 入, 因此地震脆弱性评价环节也需要 PRA 人员提出 需求, 力学结构专家提供数据信息。

c) 破坏风险与系统定量化结果: 从自然灾害风 险分析原理中可以看到破坏风险 $P(D)$ 是致灾因子、 易损性评价以及孕灾环境的函数; 核电站系统定量 化结果 (CDF/LERF) 是在危害性曲线和脆弱性评价 作为输入的基础上, 结合以事件树/故障树为工具的 电站状态分析得到风险定量结果。因此, $P(D)$ 与堆 芯损坏频率 CDF (早期大量放射性释放频率LERF）基本等价。CDF/LERF 由核电站风险分析 PRA 分析人员用软件建模获得。 d) 自然灾害基本原理的损失风险分析与核电站 的风险评估: 自然灾害基本原理中损失风险通常包 括人员伤亡损失和经济损失。而在核电领域, 目前 国内外核电站绝大多数只可以量化人员死亡损失风 险, 即每年死亡人数 (集体风险) 或年人均死亡率 (个人风险)，这一方面因为核电目前关注核安全 目标, 另一方面由于技术模型发展的限制。因此与 自然灾害基本原理中的损失风险相比, 范围较小。 此外, 核电站中的纵深防御、三级应急响应体系的 方法属于自然灾害基本原理中的减灾对策的范畴。

\section{3. 自然灾害风险分析原理在核电站自然事件 PRA 中的借鉴}

自然灾害危害性分析和 SSC 的脆弱性评估是核 电站自然事件 PRA 模型不可或缺的输入环节。基于 自然灾害风险分析的基本原理与自然事件 PRA 的联 系, 以下将探讨其在核电站自然事件 PRA 中的借 鉴:

\section{（1）自然灾害危害性分析}

自然灾害危害性分析给出的年超越频率等参数 一方面是特定核电站外部灾害篮选的重要依据, 即 当自然事件频率低于定量筛选准则, 且其后果不会 显著影响核电站安全时, 对此自然事件可以不进行 PRA 分析。另一方面, 纳入核电站外部灾害分析的 事件, 其年超越频率及其不确定度是 PRA 模型的重 要参数。目前核电站自然事件 PRA 需要各种自然灾 害在各种强度下的始发频率, 但是自然灾害危害性 分析中存在统计样本较少, 统计数据回归外推时不 确定度大等问题。模糊集方法是处理不完备信息条 件下风险分析常用的方法, 借鉴自然灾害风险评价 理论中的信息分配方法, 计算超越概率及其模糊风 险 $\pi_{m}(p)^{[3]}$, 并理解建立模糊风险和 PRA 模型的接 口, 可以补充完善核电站部分自然灾害危害性分析 结果。

（2）SSC 自然灾害脆弱性评估

核电站 SSC 的脆弱性评估的结果是 PRA 模型中 自然灾害造成的始发事件及基本事件的条件失效概 率的输入。目前国内核电站 SSC 的脆弱性评估处在 PRA 分析人员给出 SSC 的脆弱性评估的概率需求, 联合地震工程、结构力学等方向专家建立核电站 SSC 脆弱性模型及联合相关试验机构进行分析验证 阶段。核电站 SSC 的脆弱性评估一方面可以借鉴更 多的参数作为脆弱性评价的输入参数, 如从单一的 地震动参数过渡到反应谱参数等 ${ }^{[15]}$, 另一方面可以 借鉴其它领域的脆弱性分析中的经验法、解析/力学 等分析方法及破坏准则等, 加快我国核电站 SSC 自 然灾害的脆弱性评估工作。 
（3）核电站的风险评估

核电站的风险定义为后果与造成这种后果的事 故发生的频率的乘积, 即 $R($ 后果 $/$ 单位时间 $)=P($ 事 件/单位时间 $) \times C$ (后果/事件)。目前国内外核电站用 三级 PRA 模型可以量化人员死亡损失风险, 还没有 考虑经济损失和人员辐照损伤损失, 以及放射性物 质泄漏造成的长期的环境影响损失。此方面可以充 分借鉴自然灾害的基本原理及实践, 在自然灾害危 害性分析、脆弱性评估和系统定量化基础上, 联合 经济、环境、社会学方面的专家对核电站的综合损 失风险进行研究, 以全面评价核电站的风险。

\section{(4) 风险区划图}

值得注意的是, 自然灾害基本原理中的风险区 划图的概念方法在核电站 PRA 评价风险还没有, 可 以尝试其应用于核电站 PRA 的风险评价中。风险区 划图是以地图的形式表述自然灾害风险大小及其区 域分异规律的一种方式, 也是自然灾害风险评价的 终极产品 ${ }^{[16]}$ 。风险分析结果通过恰当的制图表达了 度量的风险信息，并支持决策的有效性。一方面， 核电各厂址的自然灾害危害性分析可以借鉴风险区 划图的分析结果, 另一方面, 风险区划图的概念可 以用于特定厂址的风险描述，即在 PRA 的定量分析 结果基础上, 使用重要度参数, 画出特定厂址的风 险区划图, 以直观描述在某种自然灾害下, 电厂内 各系统设备的风险分布情况。但是在这一可能的应 用过程中，需要注意通常的地震区划图作为国标, 其对象为民用建筑，基本地震动参数的概率水平为 50 年超越概率 $10 \%$ ，与核电站抗震设计基准的概率 水平-不低于年平均超越概率 $1 \mathrm{e}^{-4}$ 之间存在差异。而 且区划图的比例尺在 1:100 万的数量级上, 而核电 站概率地震危害性分析，在厂址周边 $5 \mathrm{~km}$ 范围内, 其地质调查结果的比例尺为 1:5000。因此二者的风 险区划图在工作内容、工作深度上将存在较大差 异。

\section{5. 展望}

PRA 自上世纪七十年代提出, 历经近四十年的 发展, 目前已成为与确定论方法互为补充风险评价 方法。我国核安全法规 HAF102《核动力厂设计安 全规定》5.9 中规定 “必须对核动力厂设计进行安全 分析, 在分析中必须采用确定论和概率论分析方 法”。2010 年 2 月,我国国家核安全局正式发布了 《概率安全分析技术在核安全领域中的应用》，提 出了八条技术政策以鼓励 PRA 的应用研究工作。

我国核电站 PRA 目前已在不同程度上完成了内 部事件的一级概率安全分析, 部分核电站初步开展 了二级 PRA 和外部事件 PRA 的研究, 三级 PRA 的 研究初步展开。我国是易于受灾的国家, 且具有灾
害类型多样、影响大和分布区域广等特征，因此国 内核电站外部事件的 PRA 工作需要进一步深入研 究。

基于自然事件 PRA 和自然灾害基本原理方法的 关键技术要素的联系，核电站自然事件 PRA 可以充 分借鉴自然灾害基本原理中致灾因子风险分析、承 灾体易损性评价、损失风险、风险区划图等方法和 概念, 并且与各灾种的专家、防灾减灾工程师、经 济学家和社会学家充分合作交流, 合理评价核电风 险，提高核电应对自然灾害的能力。同时核电领域 的纵深防御、三级应急响应体系、PRA 故障树等方 法可以在自然灾害风险分析中应用。

综上，核电站自然事件 PRA 的危害性、脆弱性 和损失分析是电厂风险评价的重要环节。目前国内 核电站的自然灾害风险分析尚处于起步阶段，亟需 加强与各种自然灾害研究机构的联系, 推进自然灾 害风险分析的致灾因子风险，危害性、易损性、损 失分析、风险区划图等技术在核电站自然事件 PRA 中的应用, 进一步完善核电站自然事件风险评价技 术及全范围 PRA 的应用工作。

\section{附加注解}

在国内和 IAEA, 通常称 PRA 为概率安全评价 (Probabilistic Safety Assessment, PSA)。

\section{致谢}

感谢张佳佳和荆旭对本文内容的有益讨论和建议。

\section{参考文献}

[1] IAEA International Fact Finding Expert Mission of the Fukushima DAI-ICHI NPP Accident Following the Great East Japan Earthquake and Tsunami. Mission Report. 2011.

[2] USNRC, Reactor Safety Study: An Assessment of Accident Risks in U.S. Commercial Nuclear Power Plant, WASH-1400 NUREG 75/014 .Oct. 1975.

[3] 黄崇福, 自然灾害风险评价: 理论与实践 $[\mathrm{M}]$, 科学出 版社, 2005.

[4] 张继权, 李宁, 主要气象灾害风险评价与管理的数量 方法及应用 $[M]$, 北京师范大学出版社, 2007.

[5] 庞西磼, 黄崇福, 赵思健, 自然灾害风险分析方法与相 关技术的研究进展跟踪[C],中国视角的风险分析和危 机反应-中国灾害防御协会风险分析专业委员会第四 届年会论文集, 2010.

[6] 张继权, 冈田宪夫, 多多纳裕一, 综合自然灾害风险管 理一全面整合的模式与中国的战略选择 [J], 自然灾害 学报, 15(1) (2006) 29-37.

[7] 黄崇福, 自然灾害风险分析的基本原理[J], 自然灾害 学报, 8(2) (1995) 21-30. 
[8] 陈捷飞, 郭建兵.概率安全分析方法综述 $[\mathrm{J}]$, 大亚湾 核电-核电概率安全评价技术专刊, (2006) 18-24.

[9] M. K. Ravindra, Seismic individual plant examination of external events of US nuclear power plants: insights and implications, Nuclear Engineering and Design, 175 (1997) 227-236.

[10] Standard for Level 1/Large Early Release Frequency Probabilistic Risk Assessment for Nuclear Power Plant Applications, ASME/ANS RA-Sa-2009.

[11] R. P. Kennedy, M. K. Ravindra, Seismic for nuclear power plant risk studies, Nuclear Engineering and Design, 79 (1984) 47-68.

[12] NRC, Handbook of Nuclear Power Plant Seismic Fragilities, Seismic Safety Margins Research Program, NUREG/CR-3558.

[13] J. H. Kim, I. K. Choi, J. H. Park, Uncertainty analysis of system fragility for seismic safety evaluation of NPP, Nuclear Engineering and Design, 241 (2011) 2570 2579.

[14] American Nuclear Society and National Institute of Electrical and Electronic Engineers, PRA Procedures Guides: A Guide to the Performance of Probabilistic Risk Assessment for Nuclear Power Plants, NUREG/CR-2300.

[15] 于晓辉, 吕大刚, 王光远, 土木工程结构地震易损性分 析的研究进展[C], 第二届结构工程新进展国际论坛, 2008.

[16] 张俊香, 黄崇福, 自然灾害软风险区划图模式研究 [J], 自然灾害学报, 14(6) (2005) 20-25. 